\title{
Los agujeros de la red: conocimiento, totalidad, interpretación
}

objetivo de este trabajo es discutir críticamente el artículo que, con el título de "La metodología de la investigación y los límites del conocimiento humano" publicó Felipe Martínez Rizo en el primer número de esta misma revista. ${ }^{1}$ En una breve introducción comentaré algunos aspectos de la discusión entre cuantitativistas y cualitativistas, para pasar después a exponer una concepción del conocimiento que, al diferenciar ontología de epistemología, precisa los alcances y los límites del conocimiento humano. Lo anterior me dará las bases para hablar de un concepto de totalidad construida que va más allá de lo que Martínez Rizo acepta en su artículo, para terminar con la exposición de una concepción de la metodología cualitativa como interpretación, de su necesidad, de sus limitaciones y de sus diferencias con la metodología cuantitativa.

\section{INTRODUCCIÓN: LAS DISCUSIONES "CUANTI-CUALI"}

En su libro El oficio de investigador educativo, ${ }^{2}$ en el capítulo tercero, Martínez Rizo expone un interesante recuento del desarrollo

1. Felipe Martínez Rizo, "La metodología de la investigación y los límites del conocimiento humano", Caleidoscopio, núm. 1, enero-junio de 1997, pp. 95-111.

2. Felipe Martínez Rizo, El oficio de investigador educativo, Aguascalientes: UAA, 1997. 
de las polémicas entre cuantitativistas y cualitativistas en las ciencias del hombre, remontándose al siglo XVII y terminando con la proyección del desarrollo de la polémica en el siglo XXI. En la exposición se pueden constatar las estrategias de uno y de otro bando para descalificar la postura del adversario, desde planteamientos teóricos muy elaborados hasta la diatriba pura y simple. Lo que este recuento no explicita, aunque lo deja ver, es que una de las estrategias usadas con frecuencia consiste en resaltar los puntos más débiles (que muchas veces son los más exagerados) de la posición contraria, para atacarlos con mayor facilidad. Tal es el caso, por ejemplo, cuando los cuantitativistas atacan el concepto de totalidad, interpretándolo como totalidad empírica, cuando es evidente, por una parte, que los investigadores más serios de ambas partes comparten la idea de que es imposible saber todo sobre algo y, por otra, que de los dos lados encontramos autores que critican ciertas investigaciones porque les faltó explicar algún aspecto del fenómeno bajo estudio, lo que implica que comparten un concepto de totalidad entendida como totalidad empírica, así como el criterio de que para que una investigación sea válida debe explicar todo, o no debe faltarle explicar algún aspecto (modalidad del holismo empirista más extendida), del fenómeno en cuestión.

Tiene razón el artículo que comento cuando dice que en la polémica entre cuantitativistas y cualitativistas en las ciencias del hombre, "las cosas son más complejas de lo que parecen", aunque después acepta el riesgo de continuar simplificando, no solamente al distinguir "dos maneras de concebir el conocimiento: una analítica y otra sintética" sino también al asimilarlas de alguna manera a la oposición cuanti-cuali. ${ }^{3}$

Este artículo se orienta en la dirección de dar un paso para encarar la complejidad del problema de las relaciones entre la meto-

3. Felipe Martínez Rizo, "La metodología de la investigación y los límites del conocimiento humano", Op. cit., pp. 95 y 96 
dología cualitativa y la cuantitativa corriendo, seguramente, otro riesgo: el de que la complejidad rebase las cualificaciones del que esto escribe para continuar el debate más allá de los planteamientos iniciales que aquí se exponen.

\section{UNA CONCEPCIÓN DEL CONOCIMIENTO COMO MAPA DE LA REALIDAD}

Aunque se trata de dos metáforas, la concepción del conocimiento como red o como mapa, la primera utilizada por Martínez Rizo y la segunda por mí, implican una diferencia que no es banal. La primera supone que el conocimiento es un instrumento, más o menos fino, que se arroja para capturar elementos que se encuentran como "tales" elementos, independientemente de la red, en el mar de la realidad (peces más grandes o más chicos, de una o de otra especie). La segunda supone una concepción del conocimiento como representación de una realidad que se acepta como existente independientemente de su representación, pero que para ser conocida requiere de esta última como de un mapa que, por muy detallado que sea, necesariamente simplifica la realidad y que sólo así, simplificándola, puede ser considerado como instrumento de conocimiento. Como lo dice Lévi-Strauss en Lo crudo y lo cocido, en el apartado que titula "Interludio de lo discreto", sólo empobreciendo la realidad empírica (como lo hacen el conocimiento mítico y el conocimiento científico) se puede enriquecer el conocimiento. ${ }^{5}$

Esta concepción del conocimiento se basa en una distinción fundamental entre la realidad de la teoría y otras realidades, y en la relación epistemológica entre la teoría y su objeto, buscando mantener los campos de la ontología y de la epistemología como campos que son distintos aunque, por estar estrechamente relacionados, frecuentemente se les confunda. Esto lleva a la concepción del

4. Ibidem.

5. Claude Lévi-Strauss, Lo crudo y lo cocido, México: FCE, 1968, 55-61. 
conocimiento como representación construida, y al rechazo de la reificación de los conceptos (los peces pequeños o grandes que capturaría la red) que aparece repetidamente en las ciencias sociales. El objeto del conocimiento y el método se conciben como dependientes de la teoría, y el debate entre las comunidades científicas, más que la verificación o la falsificación, como el medio para la legitimación intersubjetiva de las teorías científicas.

Mi punto de partida es el supuesto ontológico de que existen realidades que son independientes del conocimiento. No se me escapa que este supuesto es problemático, especialmente si se toman en cuenta las realidades abstractas, no materiales. ${ }^{6}$ Sin embargo, este punto no se presenta aquí como un axioma que no necesite demostración, sino, precisamente, como un punto de partida, admitiendo que en otros contextos teóricos es una problemática central. Como punto de partida me sirve para hacer las siguientes afirmaciones:

(1) El conocimiento no es idéntico a la realidad, por lo que ninguna teoría puede pretender agotar el conocimiento de la realidad.

En la investigación concreta, ni los individuos ni los equipos de investigadores pueden estudiar la entera realidad de un objeto empírico. No sólo porque no tienen los recursos técnicos adecuados, ${ }^{7}$ o por la cuestión práctica de que nunca hay tiempo suficiente, ${ }^{8}$ sino por la razón de que sería teóricamente imposible. ${ }^{9}$ Por lo tanto, mientras que es pertinènte criticar una investigación porque la teoría general en la que se basa no considera algunos elementos del

6. Ver: Rudolf Carnap, "Empiricism, Semantics and Ontology", in Boyd, Casper and Trout (eds.) The Philosophy of Science, Cambridge, Mass.: Massachusetts Institute of Technology., 1991, pp. 85-97.

7. Claude Lévi-Strauss, Antropología Estructural, Buenos Aires: Eudeba, 1970, p. 394.

8. Raymond Boudon, L’idéologie: ou l'origin des idées reçues, Paris: Fayard, 1986, p. 171.

9. Andrew Tudor, Beyond Empiricism. Philosophy of Science in Sociology, London: Routledge and Kegan Paul, 1982, p. 20. 
objeto que se piensa que son relevantes para su conocimiento, no tiene sentido criticar una investigación concreta porque no considera todos los elementos, o le falta considerar algún elemento, del fenómeno bajo estudio.

(2) El conocimiento se construye. Baso esta afirmación en lo que Tudor llama "epistemología de sentido común"10 para referirme a que muchos de los supuestos epistemológicos con los que trabajan los científicos sociales tienen un sustento confiable, aunque al mismo tiempo discutible, en el terreno de la teoría del conocimiento.

Sigo a Suppe ${ }^{11}$ en su presentación de la crítica a la posición epistemológica que llama "la posición heredada", pasando con esto de la teoría del conocimiento en general a la teoría del conocimiento científico. El autor ve esta posición como el desarrollo del positivismo lógico alemán. Según él, en el período que va de 1850 a 1880, la concepción alemana de la ciencia era una mezcla de positivismo comteano, materialismo y mecanicismo, que sostenía que: i) no hay duda de que existe un mundo real y objetivo que es independiente de los observadores individuales; ii) en ese mundo la materia es lo fundamental; iii) hay leyes mecánicas que son inherentes a las cosas mismas, y iv) la ciencia es el descubrimiento de los mecanismos de este mundo objetivo.

Desde sus primeras formulaciones se le han hecho diversas críticas a diferentes aspectos de la "posición heredada". Hacia 1870 el materialismo mecanicista fue cuestionado y la comunidad científica se movió hacia una concepción neokantiana de la ciencia. Posteriormente las escuelas de Viena y Berlín, en particular Whitehead y Rusell, influyeron en el desarrollo de la "posición heredada", llevándola a grados mayores de complejidad, particularmente desa-

10. Ibidem.

11. Frederick Suppe, "The Search for Philosophic Understanding of Scientific Theories", in Id. (ed), The Structure of Scientific Theories, Urbana: University of Illinois Press, 1974, pp. 1-242.

$\begin{array}{lllllllllllllll}C & A & L & E & \text { I } & D & O & S & C & O & P & I & O & 93\end{array}$ 
rrollando e incorporando en la teoría la lógica formal y las matemáticas. Con respecto a esto último, la exigencia de que "la teoría debe ser axiomatizada en términos de la lógica matemática (first order predicate calculus with equality)"12 fue también criticada sosteniendo que hay términos teóricos disposicionales que no pueden definirse si la teoría debe ser axiomatizada en términos de la lógica formal por lo que, o se acepta que las disposiciones no sean definidas, o se debe aceptar que la teoría pueda ser axiomatizada en términos de una lógica modal que sea capaz de expresar los subjuntivos condicionales.

Sin embargo, las críticas a la "posición heredada" y su desarrollo no modificaron lo que Suppe considera su postulado fundamental: la distinción entre teoría y datos, y la noción de verificación como criterio de cientificidad.

El verdadero reto a la "posición heredada", según Suppe, se presentó bajo dos modalidades: i) los ataques a la noción de interpretación imparcial, y la distinción entre teoría y datos, y ii) filosofías de la ciencia alternativas, que frecuentemente incluían el punto anterior. Aunque estas teorías alternativas son diferentes una de la otra en muchos detalles más o menos importantes, se pueden agrupar como sigue para los propósitos aquí enunciados:

(A) Filosofías que expresan escepticismo acerca de que puedan establecerse características comunes que compartan las teorías científicas, como es el caso, por ejemplo, de Achinstein.

(B) Filosofías basadas en el análisis de las cosmovisiones (Weltanschauungen), como las de Toulmin, Kuhn, Hanson, el primer Feyerabend y Bohm. Es preciso decir, sin embargo, que estas teorías fueron, al mismo tiempo que muy divulgadas e influyentes, mal entendidas y muy simplificadas. En particular, la teoría de Kuhn acerca de las revoluciones científicas se divulgó tanto y tan mal,

12. Frederick Suppe, The Semantic Conception of Theories and Semantic Realism, Urbana and Chicago: University of Illinois Press, 1989, p.12.

94 $\begin{array}{llllllllllllll} & C & A & \text { L } & \text { E } & \text { I } & \text { D } & O & \text { S } & C & O & \text { P } & \text { I } & O\end{array}$ 
que hizo que él mismo se lamentara de que la nociones de paradigma y de revolución científica "estaban demasiado cerca de ser lo que cada quien quisiera". ${ }^{13}$

Sigo a Kuhn en su propuesta de substituir el término de paradigma por el de ejemplares y matrices disciplinares. ${ }^{14}$ Estos términos están estrechamente relacionados con el concepto de comunidad científica, como lo estaba el de paradigma, pero el mismo Kuhn hace una autocrítica acerca de su concepción, que califica como circular, puesto que había definido una por la otra al paradigma y a la comunidad científica. Ahora propone, por tanto, reconocer que las comunidades científicas tienen una existencia independiente de las nociones de ejemplar o de matrices disciplinares, por lo que las define, sociológicamente, no sólo como constituidas por los practicantes de una especialidad científica, sino como un grupo que está unido porque tiene elementos comunes en su educación, porque se ven a sí mismos y son vistos por los demás como una comunidad, porque hay una relativa plenitud de comunicación dentro del grupo, etc. En este contexto es en el cual debe ubicarse la definición de matriz disciplinar. Es disciplinar porque es la posesión común de los que practican una disciplina profesional; es matriz porque está compuesta de elementos ordenados de varios tipos. A los ejemplares, que con las generalizaciones simbólicas y los modelos constituyen una matriz disciplinar, los define como soluciones concretas de los problemas. Llegamos así a lo que me interesa enfatizar. Es, dice Kuhn, con el uso de ejemplares como los científicos desarrollan una relación de similitud o de semejanza, lo que es usado para modelar la aplicación de generalizaciones simbólicas a nuevas situaciones experimentales; $y$ es a través de la adquisición de estas relaciones de similitud como las generalizaciones teóricas pueden

13. Thomas S. Kuhn, "Second Thoughts on Paradigms", in Suppe, F. (ed) The Structure of Scientific Theories, Op. cit., pp. 459-517.

14. Ibidem. 
llegar a tener contenido empírico, y no a través de las reglas de correspondencia entre teoría y datos como sostenía la "posición heredada" (metáfora de la red).

Hay que recordar, sin embargo, que el hecho de que la metáfora del mapa tenga una base confiable en la filosofía de la ciencia no quiere decir que sea unánimemente aceptada. Las teorías basadas en las cosmovisiones (Weltanschauungen) han sido criticadas porque emplean diferentes versiones de las siguientes tesis: i) la observación está influenciada por la teoría; ii) los significados son dependientes de la teoría, y iii) los datos dependen de la teoría. Citaré literalmente a Suppe con respecto a este último punto porque pienso que es muy importante, ya que parece implicar una concepción subjetivista de la ciencia:

La tesis (3) sostiene que (i) lo que es considerado como dato es determinado por la Weltanschaunung asociada a una teoría; (ii) que no hay conjuntos de datos neutrales que sirvan para juzgar la validez relativa de dos teorías rivales. y que (iii) la validez de una teoría debe ser juzgada de acuerdo a estándares fijados por su Weltanschaunng asociada. ¿ No quiere decir, entonces, que la tesis (3) sostiene que hay diferentes hechos para las diferentes teorías? Si la respuesta es afirmativa, ¿no quiere esto decir que la comprobación de las teorías es circular, puesto que cada teoría es sosteriida por sus propios datos? ${ }^{\text {?5 }}$

Creo que no puede responderse a estas interrogantes mientras sigamos atrapados (¿por la red?) en una concepción de la cientificidad basada únicamente en la relación entre la teoría y los datos. Más adelante introduzco la noción de cientificidad entendida como la discutibilidad de las teorías entre las comunidades científicas.

(C) Las teorías semánticas, como la del mismo Suppe. Este enfoque fue introducido hacia 1930 por A. Tarski, y fue después desarrollado por Beth, Suppes, van Fraasen y Suppe y, de una manera

15. Frederick Suppe, "The Search for Philosophic Understanding of Scientific Theories", Op. cit., p. 208.

$96-$ C 
diferente porque introdujeron reglas de correspondencia, por Sneed, Stegmuller y Moulines. ${ }^{16}$

Para Tarski, el problema de una definición satisfactoria de la verdad debe alejarse de la visión aristotélica de que la verdad de una afirmación consiste en su adecuación (o correspondencia) con la realidad, para acercarse a la propuesta de una definición semántica que establece que una afirmación es verdadera si es satisfecha por todos los objetos, y falsa si no, entendiendo la satisfacción como la relación entre objetos arbitrarios y ciertas expresiones.

De acuerdo a Suppe:

La concepción semántica toma su nombre del hecho de que construye teorías acerca de a qué se refieren sus formulaciones cuando se les da una interpretación semántica (lógica) [...] En la concepción semántica, el corazón de una teoría es una estructura teórica extralinguística. ${ }^{17}$

Varias versiones de esta concepción coinciden en afirmar que "los fenómenos del mundo real (o un fenómeno particular del mundo real) establecen una relación de mapeo (subrayado mío) con la estructura teórica". ${ }^{18}$

Nuevamente hay que decir, sin embargo, que los autores difieren acerca de la naturaleza de esta relación. De acuerdo a Suppe, el empiricismo constructivista de van Fraasen sostiene un antirealismo porque rechaza la idea de que las teorías son historias verdaderas sobre cómo es el mundo, aunque la aceptación de una teoría implica la creencia de que es verdadera. ${ }^{19}$ Putnam sostiene lo que llama un "realismo interno", ${ }_{20}$ y Suppe propone un cuasi-realismo que él

16. Frederick Suppe, The Semantic Conception of Theories and Semantic Realism, Op. cit.

17. Ibid., p.4.

18. Ibidem.

19. Ibidem.

20. Hilary Putnam, Realism and Reason. Philosophical Papers. Volume 3, Cambridge: Cambridge University Press, 1983. 
mismo asimila a la afirmación antirealista de van Fraasen, enfatizando que esta versión de la concepción semántica no emplea la distinción teoría/datos de la "posición heredada". Por mi parte, sin meterme en la controversia, sólo puedo decir con Tudor que:

...ya sea que se basen (esas relaciones) en la analogía o en la homología de las estructuras, 0 en cualquier otra de las muchas posibilidades exploradas en la literatura, las teorías son invenciones (subrayado mío) que usamos para dar sentido a algo que creemos que conocemos. ${ }^{21}$

A pesar de que Suppe no deja de señalar las controversias, uno puede derivar de sus escritos la impresión de que la teoría del conocimiento científico de alguna manera converge hacia las teorías basadas en las cosmovisiones (Weltanschauungen) y hacia las teorías semánticas.

Pero basta leer los artículos sobre epistemología y sobre filosofía de la ciencia contenidos en los volúmenes editados, respectivamente, por Goodman y Snyder ${ }^{22}$ y por Boyd, Gasper y $\operatorname{Trout}^{23}$ (1991) para darse cuenta de que la situación es muy diferente. Goodman y Snyder, por ejempln, incluyen la teoría de Quine acerca de una epistemología naturalizada (naturalized) en su último capítulo acerca de los enfoques alternativos. Boyd, Gasper y Trout, en el ensayo introductorio al libro que editan afirman que los principales contendientes entre los enfoques generales sobre la filosofía de la ciencia son el realismo científico, el constructivisno neokantiano y versiones post-positivistas muy sofisticadas del empirismo.

Regresando a nuestra epistemología de sentido común (fundamentada, aunque discutible y discutida), y al terreno, más familiar

21. Andrew Tudor, Beyond Empiricism. Philosophy of Science in Sociology, Op. cit., p. 112.

22. Michel Goodman and Rober A. Snyder (eds), Contemporary Readings in Epistemology, Englewood Cliffs: Prentice Hall, 1993.

23. Richard Boyd, Philip Gasper y J.D. Trout (eds), The Philosophy of Science, Cambridge, Mass.: Massachusetts Institute of Technology, 1991. 
para mí, de las ciencias sociales, refrendo mi concepción de que los objetos del conocimiento científico se construyen teóricamente. ${ }^{24}$ Conocer no es ver (ni pescar). El conocimiento objetivo de la ciencia presupone siempre una teoría. ${ }^{25}$ Como lo dice Lévi-Strauss, "la explicación comienza cuando hemos logrado construir el objeto" ${ }^{26}$ Este acto de construcción ha sido interpretado como una acto de ruptura, porque el conocimiento científico se concibe como la interpretación de otras interpretaciones. ${ }^{27}$ Lo que llamamos datos son interpretaciones ${ }^{28}$ Como intenta demostrarlo Feyerabend, el término masa, por ejemplo, tiene dos significados diferentes e incompatibles en la mecánica clásica y en la teoría de la relatividad. ${ }^{29}$

Sin embargo, si la teoría del conocimiento científico no se concibe como un modelo forzoso al que la práctica de los científicos deba ajustarse para ser considerada ciencia, sino como el análisis de esa práctica tal como de hecho se lleva a cabo ${ }^{30}$ hay que reconocer que muchas teorías científicas, o al menos muchas teorías de las ciencias sociales, consideran los datos como medios para probar las teorías. Pero esto no siempre implica una concepción empirista del conocimiento científico. En palabras de Bourdieu, "la única manera de pensar correctamente es a través de datos empíricos teóricamente construidos". ${ }^{31}$

24. Pierre Bourdieu, Jean-Claude Chamboredon y Jean-Claude Passeron, El oficio de sociólogo. Presupuestos epistemológicos, México: Siglo XXI, 1975, pp. 27-82.

25. Talcott Parsons, La estructura de la acción social, Madrid: Guadarrama, 1968, p. 14.

26. Claude Lévi-Strauss, Savage Mind, London: Weidenfeld and Nicolson, 1966, p. 362.

27. Pierre Bourdieu, Jean-Claude Chamboredon y Jean-Claude Passeron, Op.cit., pp. $27-50$.

28. Jean Piaget, Tratado de lógica y conocimiento científico. Tomo I, Buenos Aires: Paidos, 1977, p. 20.

29. Frederick Suppe, "The Search for Philosophic Understanding of Scientific Theories", Op. cit.

30. Ibidem.

31. Pierre Bourdieu, Réponses. Pour une anthropologie réflexive, Paris: Seuil, 1992, p.135. 
Sostengo también que los métodos son dependientes de la teoría, concebidos como ars inveniendi, no como ars probandi. . $^{32}$ Por método entiendo las estrategias o reglas de la investigación, que son diferentes de la epistemología, de la teoría del conocimiento científico y de las técnicas. Coincido con Merton cuando critica a los empiristas por confundir la recolección de información con la recolección de observaciones científicamente pertinentes. ${ }^{33}$ No acepto, pues, la idea de la investigación determinada por el método. En palabras de Bourdieu, Chamboredon y Passeron:

Frente a algunas investigaciones concebidas en función de la causa lógica 0 metodológica, no puede sino evocarse, con Abraham Kaplan, la conducta de un borracho que, habiendo perdido (en otro lado -paréntesis mío-) la llave de su casa, la busca sin embargo con obstinación bajo la luz de un farol, ya que alega que alli se ve mejor. ${ }^{34}$

Concibiendo lo’s objetos de la ciencia como construidos teóricamente, y la metodología como dependiente de la teoría, ¿debo concluir que cualquier procedimiento es válido, o concebir la empresa científica como especulaciones ingeniosas y conclusiones impresionistas, como reprocha Merton a los teóricos globales ${ }^{235}$ De acuerdo a Chalmers, ${ }^{36}$ algunos (mal)intérpretes de la obra de Feyerabend titulada Against Method ${ }^{37}$ responderían afirmativamente. Por mi parte, yo sostengo que las teorías son un mapa de la realidad. Y tomo de las teorías de las cosmovisiones (Weltanschauungen) la concepción del consenso y el debate entre las comunidades científicas co-

32. Pierre Bourdieu, Jean-Claude Chamboredon y Jean-Claude Passeron, Op. cit., p.17.

33. Robert K Merton, Teoría y estructura sociales, México: FCE, 1964, p. 438.

34. Pierre Bourdieu, Jean-Claude Chamboredon y Jean-Claude Passeron, Op. cit., p. 21.

35. Robert K Merton, Op. cit., p. 437.

36. A. F. Chalmers, What is this Thing Called Science?, Milton Keynes: Open University Press, 1982, p. 134.

37. Paul Feyerabend, Against Method: Outline of an Anarchistic Theory of Knowledge, London: New Left Books,1975.

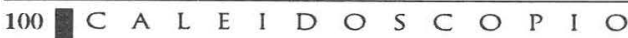


mo sustituto de la correspondencia entre teoría y datos. Concibo la claridad teórica y el uso de la lógica formal como medios para hacer que las teorías sean discutibles, y la discutibilidad como elemento fundamental de la cientificidad. Como lo dicen Bourdieu, Chamboredon y Passeron en un pasaje previo del mismo párrafo donde citan al borracho de Kaplan:

No se trata aquí de negar que la formalización lógica encarada como medio para poner a prueba la lógica en acto de la investigación y la coherencia de sus resultados constituye uno de los instrumentos más eficaces de control epistemológico. ${ }^{38}$

Vale la pena citar la epistemología evolucionista de Popper, ${ }^{39}$ que rechaza el observacionismo (¿la pesca?), al que llama el barril teórico de la mente, proponiendo, en cambio, la reflexividad del lenguaje como un medio de vigilancia epistemológica:

Para hacer posible que una teoría pueda ser criticada, el organismo debe poder ver la teoría como objeto [...] De esta manera nuestras teorías, nuestras conjeturas, nuestros ensayos o nuestros ensayos y errores pueden convertirse en objetos; objetos como las estructuras físicas, vivas o muertas. Pueden convertirse en objetos de investigación crítica. ${ }^{40}$

Es decir, la teoría puede convertirse en objeto de debate entre las comunidades científicas.

De acuerdo a Giménez Montiel, ${ }^{41}$ la objetivación metodológica permite la esencial discutibilidad de las teorías científicas. Es algo

38. Pierre Bourdieu, Jean-Claude Chamboredon y Jean-Claude Passeron, Op. cit., p. 21. 39. Karl R. Popper, "Evolutionary Epistemology", in Goodman and Snyder (eds) Contemporary Readings in Epistemology, Op. cit., pp. 338-350.

40. Ibid., p. 346.

41. Gilberto Giménez Montiel, "La problemática de la cultura en las ciencias sociales", en Id. (ed) La teoría y el análisis de la cultura, México: SEP-U de G-COMECSO, 1987, pp. 15-72. 
similar a la vigilancia epistemológica en la comunidad científica de la que hablan Bourdieu, Chamboredon y Passeron:

...la eficacia científica de la crítica depende de la forma y estructura de los intercambios en los cuales se cumple: todo induce a considerar que el intercambio generalizado de críticas donde, como en el sistema de intercambios matrimoniales del mismo nombre, $\mathrm{A}$ critica a $\mathrm{B}$ que criticaría a $\mathrm{C}$ que criticaría a $\mathrm{A}$, constituye un modelo más favorable para una integración orgánica del medio científico que, por ejemplo, el club de admiradores mutuos como intercambio restringido de buenos procedimientos. ${ }^{+2}$

(3) Una consecuencia de los dos puntos anteriores es que se debe estar alerta para evitar el muy frecuente error de reificar los conceptos teóricos. Parsons, por ejemplo, señala la confusión que hace que se identifique un sistema teórico general lógicamente cerrado, con un sistema empíricamente cerrado. ${ }^{43}$ Y Bourdieu, hablando de las reglas de un juego que son diferentes de los modelos construidos por los científicos para explicarlo, dice:

Yo pienso que si se olvida esa distinción, se corre el riesgo de cometer uno de los más desastrosos errores que pueden producirse en las ciencias humanas, aquel que consiste en confundir, en la bien conocida frase de Marx, "las cosas de la lógica con la lógica de las cosas".

El mismo autor, refiriéndose a los modelos de simulación y a los modelos de las prácticas sociales, a los que reconoce su función heurística, previene a los científicos sobre el peligro de caer en la tentación de convertir los modelos de la realidad en la realidad del modelo. En otras palabras, los teóricos deben evitar "la mayor fala-

42. Bourdieu, Pierre, Jean-Claude Chamboredon y Jean-Claude Passeron, Op. cit., pp.108-109.

43. Taltcott Parsons, La estructura de la acción social, Madrid: Guadarrama, 1968.

44. Pierre Bourdieu, The Logic of Practice, Stanford: Stanford University Press, 1990, p. 61.

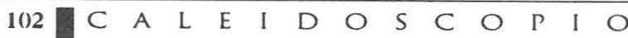


cia, aquella que consiste en poner en las cabezas de las gentes que están estudiando lo que ellos, los teóricos, deben tener en su cabeza para entender lo que hace la gente". ${ }^{45}$

\section{LA TOTALIDAD COMO CONCEPTO CONSTRUIDO}

Coincido con Martínez Rizo ${ }^{46}$ cuando afirma que una totalidad que signifique empíricamente todo no puede ser cognoscible, ni analítica ni sintéticamente, ni científica ni intuitivamente. Viene al caso un cuento titulado "El punto", de un autor mexicano cuyo nombre no recuerdo. Se trata de un estudioso que, desesperado porque ningún tema que trataba podía quedar agotado, se puso a buscar un tema que fuera tan pequeño y tan simple que pudiera decirse todo de él, y ser el primer estudioso que lograra decir todo de algo. Creyó encontrar este tema en el punto, algo tan pequeño y tan simple que debería ser posible conocer y decir todo de él. Se puso a trabajar y a estudiar: el punto decimal... el punto y seguido en la literatura alemana del siglo XIX.... el arte del punto de cruz... la distancia más corta entre dos puntos... los puntos de la acupuntura china... hasta que murió sin haber agotado el tema.

Cuando hablan de totalidad, algunos investigadores más serios, cuantitativistas y cualitativistas, no se refieren a un todo empírico sino a una totalidad teóricamente construida. Esto lo señala ya Martínez Rizo en su artículo. Yo quiero ir, sin embargo, un poco más allá exponiendo un concepto de totalidad construida que se concibe como algo más, algo diferente de la suma de sus partes. Para hacerlo voy a recurrir a exponer algunas concepciones de totalidad que, aunque son diferentes entres sí, comparten esa idea de totalidad como algo más que la suma de sus partes.

45. Ibid., p.80

46. Felipe Martínez Rizo, "La metodología de la investigación y los límites del conocimiento humano", Op. cit.

$\begin{array}{llllllllllllll}C & \text { A } & \text { L } & \text { E } & \text { I } & \text { D } & O & S & C & O & \text { P } & \text { I } & O & \mathbf{1 0 3}\end{array}$ 
El lingüista danés Hjemslev, fundador de la teoría glosemática, parte de la afirmación de Saussure de que la lengua es ante todo forma, para sacar y sostener unas consecuencias extremas de esta afirmación, a las que Saussure no llegó. Si la lengua es forma, no hay, entonces, nada de positivo en el lenguaje en el sentido de no poder recurrir a la significación o a la realidad, a la substancia, para definir los elementos del lenguaje. No se puede recurrir ni siquiera a la oposición, pues esta supone la definición positiva de los términos. Según Ducrot, Hjemslev sostiene que: "la unidad (del lenguaje), puramente racional y negativa, no puede definirse en sí misma -lo único importante es el simple hecho de su diferencia con respecto a las demás-, sino únicamente por las relaciones que la unen a las demás unidades de la lengua." Y Malmberg añade:

Una de las principales metas del análisis es, según Hjemslev, describir las diferentes dependencias (relaciones) mutuas que existen entre las partes del texto. Considera que el objeto analizado sólo puede definirse como un todo en virtud de estos vínculos. Lo importante en el objeto analizado -el lenguaje, aquí- no es más que las relaciones entre las partes. 'Una totalidad no consiste en cosas, sino en relaciones'. No es la sustancia como tal la que es objeto de la ciencia, sólo sus nexos interiores. ${ }^{4 i}$

C. Lévi-Strauss en su obra Antropología Estructural, ${ }^{48}$ cita a Trubetskoj diciendo que el programa que éste propone para la fonología puede aplicarse en el terreno de la antropología. Dos de los puntos de este programa dicen que la fonología "rehusa tratar los términos como entidades independientes y toma como base de su análisis, por el contrario, las 'relaciones' entre los términos; introduce la noción de sistema..." ${ }^{49}$

47. Bertil Malmberg, Los nuevos caminos de la linguiística, México: Siglo XXI, 1981, p. 157.

48. Claude Lévi-Strauss, Antropología Estructural, Buenos Aires: Eudeba, 1970.

49. Ibid., p. 31 . 
Esto le sirve de base para proponer, en el capítulo Xv de la misma obra ${ }^{50}$ el "Concepto de Estructura en Etnología", cuyos elementos fundamentales son los siguientes: i) el concepto de estructura social no se refiere a la realidad empírica, sino a los modelos construidos con base en ella; la estructura social, por lo tanto, no es las relaciones sociales, sino que éstas son la materia prima empleada para la construcción de modelos que hacen manifiesta la estructura social. ii) Los modelos constituyen el objeto propio del análisis estructural. Saber en qué consisten es un problema que no es etnológico, sino epistemológico. iii) Para merecer el nombre de estructura, los modelos deben satisfacer, entre otras, las siguientes condiciones: a) una estructura presenta el carácter de sistema. Esto implica que la modificación de cualquiera de sus elementos comporta una modificación de todos los otros; b) cada modelo pertenece a un grupo de transformaciones, cada una de las cuales pertenece a un modelo de la misma familia, de manera que el conjunto de tales transformaciones constituyen un conjunto, otro sistema, de modelos.

En El totemismo en la actualidad, ${ }^{51}$ Lévi-Strauss establece que el objeto del conocimiento desde la perspectiva estructural no está formado por sus elementos, sino por sus relaciones.

En su obra acerca del estructuralismo, Jean Piaget ${ }^{52}$ dice que, aunque difieren en muchos aspectos, se pueden encontrar características comunes a los estructuralismos, que permiten definir el concepto de estructura, de una forma inductiva, en dos aproximaciones: La primera es que una estructura es un sistema de transformaciones que comporta leyes en cuanto sistema, en oposición a las propiedades de sus elementos, y que se conserva y enriquece gracias al juego mismo de sus transformaciones, sin que éstas conduzcan fuera de sus fronteras o apelen a elementos externos. En breve, una

50. Ibid., pp. 249-289

51. Claude Lévi-Strauss, El totemismo en la actualidad. México: FCE, 1965.

52. Jean Piaget, Lo strutturalismo, Milano: Il Saggiatore, 1973, pp. 37-49. 
estructura comporta tres características: totalidad, transformaciones y autoregulación. La totalidad consiste en que una estructura no puede ser un agregado. Está formada por elementos, pero éstos se subordinan a leyes que caracterizan al sistema como tal. Tales leyes de composición no se reducen a asociaciones acumulativas, sino que son propias del conjunto, distintas de las de las partes: son las leyes del sistema. La característica de transformación se deduce ae la anterior: si lo que es propio de la totalidad son sus leyes de composición, estas leyes son estructurantes por naturaleza. La autoregulación determina un cierto cierre de la estructura, lo que no quiere decir que una estructura no pueda entrar como subestructura de una estructura más amplia, con lo cual pasará a ser un elemento que sólo tiene sentido por las relaciones que se establecen con los elementos de la nueva totalidad. La segunda aproximación es que la estructura debe poder dar lugar a una formalización

De acuerdo a Bourdieu, lo que constituyó la novedad esencial del estructuralismo fue:

...la introducción en las ciencias sociales del método estructural o, más simplemente, de un modo de pensamiento relacional el cual, rompiendo con el modo esencialista de pensamiento, lo lleva a uno a caracterizar cada elemento por las relaciones que lo unen con todos los otros en un sistema del que deriva su significado y su función. ${ }^{53}$

\section{¿Cuantitativo o cualitativo?}

Para ensayar una respuesta a la pregunta, parto de la concepción que liga a la metodología cualititativa con la hermenéutica, para definirla como interpretación. Pero, apoyado en la distinción entre epistemología y ontología que me sirve como punto de partida, no

53. Pierre Bourdieu, The Logic of Practice. Stanford: Stanford University Press, 1990, p. 4.

106 C A 
comparto la idea de que la necesidad de la hermenéutica se base en una distinción ontológica entre los objetos de las ciencias naturales y los objetos de las ciencias humanas. Como lo dije arriba, concibo la metodología como dependiente de la teoría, lo que me lleva a responder la pregunta acerca de si son mejores los métodos cualitativos o los cuantitativos con un "depende": depende de la pregunta que queramos responder. Si la teoría me lleva a plantearme una pregunta acerca de cuántos votos es probable que obtenga un partido político en unas elecciones, es mejor cuantificar. Si, en cambio, me pregunto cuál sería la concepción del mundo de los habitantes de Mesoamérica antes de la conquista, es mejor interpretar.

Debo decir, sin embargo, que estoy de acuerdo con Martínez Rizo en su crítica de la intuición como método científico. Y debo admitir que, así como en la metodología cuantitativa más pobre pero, desafortunadamente, muy divulgada, un esquema de investigación se enunciaría en los siguientes, o semejantes términos: "Hipótesis, variable independiente, variable dependiente, aplicación de cuestionarios, análisis cuantitativo de los resultados", también en la metodología cualitativa se practica, con lamentable frecuencia, una interpretación que es sólo el equivalente de un "a mí se me hace que...", ignorando los desarrollos más serios, pero difíciles y laboriosos, de la metodología. Tal es el caso, por ejemplo, de muchos de los colaboradores del Journal of Popular Culture, lo que llevó a Lohof, en un análisis autocrítico, a responder a la pregunta: ¿qué métodos hemos elegido?, de la siguiente manera:

Puesto brevemente, no hemos sido muy duchos cuando pedimos prestado, ni muy ingeniosos en nuestras invenciones. En cambio, hemos robado desfachatadamente los métodos más rústicos que circulan en la academia, y hemos pretendido que eso es suficiente. ${ }^{5+}$

54. Bruce A. Lohof, "Popular Culture: The Journal and the State of the Study", Journal of Popular Culture, 6.3, 1973, 292-300, p. 457. 
Como resultado, dice Jensen:

Tales análisis no pueden, normalmente, convertirse plenamente en objetos de (des)acuerdos intersubjetivos en una comunidad cientifica o en un foro público. Más bien, la validez de una interpretación depende en una confianza universal en la sensibilidad y la experiencia del académico que las hace, en su legitimidad y autoridad, o quizá en lo original y estimulante de la interpretación. ${ }^{55}$

No toda la metodología cualitativa, sin embargo, es intuitiva, ni todos los cualitativistas proceden de la manera que critica Lohof. En la metodología cualitativa se hacen esfuerzos serios, que aquí no es el lugar para detallar, para convertir a las teorías en objetos de discusión, de (des)acuerdos intersubjetivos. En esta tarea, pienso yo, la formalización juega un papel importante.

La misma metodología de la ciencia debe ser objeto de discusión entre las comunidades académicas, pues sólo esta discusión permite avanzar en el terreno del conocimiento científico. Una de las formas de esta discusión es el debate entre cuantitativistas y cualitativistas, que será más fructífero mientras más se tomen en cuenta para la discusión los desarrollos más serios y prometedores de ambas perspectivas. 중

55. Klaus Bruhn Jensen, "Humanistic Scholarship as Qualitative Science: Contributions to Mass Communication Research", in K. B Jensen, and N.W. Jankowski (eds) A Handbook of Qualitative Methodologies for Mass Communication Research, London and New York: Routledge, 17-43, 1993, pp. 31-32.

108 C C A 